Mots. Les langages du politique

$68 \mid 2002$

Les métaphores spatiales en politique

\title{
En haut et au centre : la prééminence présidentielle
}

Eminence and centrality. The presidential pre-eminence

Arriba y al centro. El predominio presidencial

\section{Bertrand Pirat}

\section{(2) OpenEdition}

\section{Journals}

Édition électronique

URL : https://journals.openedition.org/mots/6803

DOI : $10.4000 /$ mots.6803

ISSN : 1960-6001

\section{Éditeur}

ENS Éditions

\section{Édition imprimée}

Date de publication : 1 mars 2002

Pagination : 59-72

ISBN : 2-84788-007-0

ISSN : 0243-6450

\section{Référence électronique}

Bertrand Pirat, «En haut et au centre : la prééminence présidentielle », Mots. Les langages du politique

[En ligne], 68 | 2002, mis en ligne le 29 avril 2008, consulté le 23 avril 2022. URL : http://

journals.openedition.org/mots/6803; DOI : https://doi.org/10.4000/mots.6803 


\section{En haut et au centre : la prééminence présidentielle}

La présidence de la République occupe une place particulière dans les institutions de la $\mathrm{V}^{\mathrm{e}}$ République en raison des controverses qu'elle a suscitées dès sa naissance : le président doit-il être un " président arbitre » ou le chef de l'exécutif et de la majorité ${ }^{1}$ ? Le référendum sur la durée du mandat présidentiel a remis cette question au premier plan, en articulant de manière assez singulière la question de la temporalité avec celle de la spatialité : «Pour certains, remarque Lucien Jeaume, réduire le mandat au quinquennat est un biais pour redimensionner le personnage présidentiel, qui ne doit plus être au-dessus mais en face du parlement /.../ Cette métaphore spatiale est un enjeu qui se développe en de multiples conséquences $\|^{2}$. Ses contradicteurs rétorquent qu'un président au-dessus des partis est une pure « fiction constitutionnelle ». Une fiction certes, mais dont les effets de réalité sont prégnants. En effet, les transformations récentes de l'espace public ont fait de l'élection du président de la République l'ultima ratio de la compétition politique nationale, à l'exclusion de tout autre enjeu ${ }^{3}$. La vision médiatique du jeu politique a largement accrédité la prééminence présidentielle et dès lors, le

\footnotetext{
Centre de Politologie de Lyon, CERIEP, IEP, 14 avenue Berthelot, 69000 Lyon Cedex 07.

1. Le débat normatif sur le rôle du président de la République se double d'un débat théorique sur la nature du fait institutionnel. Les disciplines juridiques posent la question du pouvoir présidentiel en termes de prérogatives inscrites dans le texte constitutionnel lui-même, alors que la science politique élargit la chaine explicative au champ politique dans son ensemble pour rendre compte de la prééminence présidentielle. Pour un aperçu synthétique des termes de ce débat, voir B. François, Le régime politique de la Ve République, La Découverte, 1998, p. 6-8.

2. L. Jeaume, «Le quinquennat et la Constitution », Esprit, $\mathrm{n}^{\circ}$ 266-267, aout-septembre, 2000 , p. 219 et suiv. (c'est nous qui soulignons).

3. À propos des effets structurants de l'institution présidentielle sur le jeu politique de la $\mathrm{V}^{\mathrm{e}}$ République, voir D. Gaxie, «Les fondements de l'autorité présidentielle. Transformations structurelles et consolidation de l'institution » dans Le président de la République. Usages et genèses d'une institution, B. Lacroix, J. Lagroye (dir.), Presses de la FNSP, 1992, p. 334-375.
} 
rapport ordinaire à la politique se trouve investi par des phénomènes de spectacularisation et de personnalisation de l'exercice démocratique ${ }^{4}$. C'est ce rapport ordinaire à l'institution présidentielle que nous voudrions saisir, plus particulièrement à travers le véritable " phénomène de librairie » qui s'est développé autour de François Mitterrand, dans l'accumulation d'ouvrages publiés à la fin du second septennat et juste après sa mort (biographies, témoignages, pamphlets, confessions, souvenirs, chroniques...). Les récits portant sur le personnage présidentiel qui s'y développent peuvent être considérés à la fois comme le résultat d'un travail antérieur de formalisation ${ }^{5}$ du rôle institutionnel et comme une modalité spécifique de ce travail. À ce double titre, ils constituent des matériaux précieux pour observer comment se dessine la prééminence de la figure présidentielle et plus particulièrement comment cette prééminence est située dans l'espace politique.

Notre corpus est constitué de quatre livres publiés entre 1995 et 1997 :

Laure Adler, L'année des adieux, J'ai lu, 1996 (première édition Flammarion, 1995) (noté ADA).

Franz-Olivier Giesbert, Le vieil homme et la mort, Gallimard, 1996 ; (noté VHM).

Régis Debray, Loués soient nos seigneurs. Une éducation politique, Gallimard, 1996 (noté LSS).

Alain Duhamel, Portrait d'un artiste, Flammarion, 1997 (noté PA).

Bien qu'adoptant des modes de présentation différents, ils peuvent être regroupés dans un genre narratif commun - les récits du personnage présidentiel - dont le principe repose entre autres (la dimension chronologique est également centrale, mais laissée de côté ici) sur un double mouvement de personnification de l'institution présidentielle et de présidentialisation de la personne. Ces ouvrages ont fait l'objet d'un relevé systématique des métaphores spatiales afférentes aux mots président, présidence, pouvoir, Élysée et François Mitterrand ${ }^{6}$.

4. Notamment lors de l'élection présidentielle. Voir P. Braud, « Élire un président... ou honorer les dieux ? », Pouvoirs, n 14, 1980, p. 15-28.

5. «Par le terme de formalisation peut être généralement désigné l'effet des processus établissant la "figure" de l'institution et conférant du sens aux pratiques qui en relèvent. S'inscrit dans cet ensemble de processus la multiplicité des discours tenus et des conduites adoptées par un grand nombre d'agents dispersés, engagés simultanément dans plusieurs sites d'interaction et dont les activités font surgir les différents aspects d'une identité institutionnelle irréductible au seul discours officiel » (B. Lacroix, J. Lagroye, "Introduction», dans Le président de la République, op. cit., p. 10).

6. Nous avons choisi de ne pas limiter le corpus aux seules métaphores relatives au président ainsi nommé car les métaphores spatiales sont employées dans un jeu de métonymies 
La métaphore n'est pas tant envisagée ici comme un procédé stylistique que comme une modalité ordinaire de connaissance du monde social qui engage autant des modes de représentation que des modes d'action ${ }^{7}$. C'est pourquoi le corpus ainsi constitué a été analysé au regard des usages de la métaphore pratiqués par les commentateurs de François Mitterrand. Pour chaque système d'expressions métaphoriques (le haut et le centre), on a distingué quatre usages de la métaphore : un usage toponymique qui consiste à nommer la présidence, un usage topographique qui la situe par rapport à un élément référent, un usage topométrique qui implique une mesure, et un usage topologique qui a pour but la production explicite d'un savoir. Après la présentation des deux systèmes de métaphores spatiales, nous poserons la question de leur compatibilité et de leur cohérence ${ }^{8}$.

\section{Le haut}

\section{Toponymie}

Pour désigner la présidence de la République, certaines expressions métaphoriques la situent en haut, et même le plus en haut :

« Il n'y a qu'un seul oubli : le nom du Président ! Le gouvernement avait omis de l'imprimer sur la couverture du livre blanc, se contentant laconiquement de l'expression : "les plus hautes autorités de l'État"» (ADA, p. 158).

« En ce jour où je prends possession de la plus haute charge » (L'auteur cite le discours de François Mitterrand du 21 mai 1981, PA, p. 129).

\section{Topographie}

Cette toponymie institutionnelle se double d'une véritable topographie. Les expressions métaphoriques fonctionnent comme une représentation graphique - mise en mots - du relief et des configurations relatives à la position du président. Cette topographie institutionnelle suppose la mise en relation du président avec un autre élément et sa

croisées entre un lieu (L’Elysée), une fonction (présider), un titre (président), une institution (la présidence), une personne (François Mitterrand) et une notion (le pouvoir).

7. Je remercie les membres du Séminaire de Lexicologie politique et de Politologie lexicale (Groupe de recherche Rhêma - CERIEP) pour leurs questions et leurs suggestions.

8. On reprend ici l'approche de la métaphore proposée par G. Lakoff, M. Johnson, Les métaphores dans la vie quotidienne, Minuit, 1985. 
projection sur un plan vertical. François Mitterrand, en tant que président, occupe alors une position de surplomb. Il est au-dessus. L'élément par rapport auquel il est positionné définit du même coup l'espace de projection. En premier lieu, le président surplombe un espace spécifiquement politique, comme l'espace partisan :

«Le Président se demande /.../ comment honorer le socialisme en se déplaçant à Liévin, tout en demeurant au-dessus des querelles du parti » (ADA, p. 225).

ou l'espace politique dans son ensemble :

«Après quoi, bien installé dans son personnage de patriarche au-dessus de la mêlée notamment grâce à deux années de cohabitation -, il a soudain retrouvé mordant et griffe pour faire de nouveau acte de candidature /.../ ( (ADA, p. 53).

ou encore un espace civique, gradué par les hiérarchies républicaines, dont le degré le plus élevé est la loi. La position du président varie alors d'un commentateur à l'autre...

« Mitterrand ne se considère pas au-dessus des lois, mais il aime bien fabriquer les lois de sa tribu, dont l'esprit peut se résumer ainsi : fidélité inconditionnelle, disponibilité totale, amour du passé » (ADA, p. 42).

« Tant que son cœur battait, son bon plaisir [de Mitterrand] aura été d'enfreindre les règles et de s'ériger au-dessus des lois autant que du genre humain » (VHM, p. 9).

Comme le laisse penser ce dernier énoncé, la position de surplomb du président n'est pas référencée seulement à un espace civique ou politique. La métaphore spatiale s'applique à l'univers des valeurs humaines et morales :

«Quand nous en restions là, il [Mitterrand] me semblait habiter au-dessus de moimême » (VHM, p. 18).

«Un des directeurs de Paris Match avait en effet /.../ contacté le Président pour l'en informer et connaitre son opinion en cas de publication : "Je suis au-dessus de tout cela", avait-il d'abord répondu à ses collaborateurs » (à propos de Mazarine, ADA, p. 246).

La position de surplomb relatif du président se transforme parfois en surplomb absolu. Notre corpus révèle la surabondance de l'adjectif $s u$ prême. Provenant du latin supremus, superlatif de superus, suprême désigne, selon un sens primaire qui relève de la spatialité, ce « qui est audessus de tous, dans son genre, dans son espèce " (Le Petit Robert, 1993). Souvent utilisé par le général de Gaulle et ses commentateurs pour qualifier le chef de l'État, il est ici repris à propos de François Mitterrand, tant pour qualifier la fonction que la personne, afin de situer 
moralement les attributs propres à une position d'éminence absolue. Suprême donc, le statut du président par rapport au premier ministre :

«Contrairement à ce qu'affirment la plupart des commentateurs de l'époque, il s'agit beaucoup moins d'affaiblir Michel Rocard que de consolider son [Mitterrand] statut personnel d'arbitre suprême de la société » (sur la gestion des conflits politiques du début des années 1990, PA, p. 145).

Suprêmes également, les qualités de la personne qui lui assurent la suprématie dans le combat politique :

«Ce que l'on a si souvent présenté comme son habileté suprême - avec présomption de cynisme et parfum de machiavélisme - relève d'abord de cette manière Mitterrand » (à propos de la «méthode Mitterrand» de conquête du pouvoir, PA, p. 106).

Le qualificatif de suprême vient désigner un espace différencié, dont la hauteur opère une césure avec ce qui relève de l'ordinaire :

« S'il créait les structures, en marges des normes ordinaires, s'il tolérait leur clandestinité, il devenait le responsable suprême de leurs agissements /.../» (à propos des écoutes téléphoniques et de la cellule anti-terroriste de l'Elysée, PA, p. 228).

« C'était un homme [Mitterrand] qui plaçait très haut sa liberté personnelle - orgueil suprême, assurément - et s'était affranchi souverainement des lois ordinaires de la morale » (VHM, p. 32).

Cet espace différencié, en tant que hauteur « encore plus haute » et presque sans limite, se révèle être un lieu d'une spécificité irréductible que seul le président occupe :

«Personne ne devient président de la République au suffrage universel par hasard ou par distraction. À ce stade de responsabilités suprêmes, il faut un appétit spécial, une conscience aigüe de ses capacités » (PA, p. 104).

« Sa force de caractère, l'importance même de ses responsabilités, la difficulté des choix politiques à faire, cette concentration propre au pouvoir suprême et à l'univers totalement atypique qu'il implique, ont-elles joué un rôle dans ce répit /.../ ( (à propos de la maladie de François Mitterrand, PA, p. 35).

Cette topographie institutionnelle révélatrice de la situation propre à l'institution présidentielle trace également des cheminements. La représentation n'est alors plus statique mais dynamique. Et lorsqu'il est question du président, cette dynamique est surtout ascensionnelle :

«C'est aussi que François Mitterrand avait, on le sait aujourd'hui, accédé à ce pouvoir suprême qu'il recherchait depuis si longtemps pour apprendre, à peine quelque mois plus tard, qu'il était frappé par une maladie assassine /.../» (PA, p. 34).

«Son [Mitterrand] ambition l'a mené au pouvoir suprême mais sa méthode l'a abusé » (PA, p. 102). 
Plus explicite encore, la métaphore de l'escalier :

«Il convoitait les responsabilités, il aspirait au pouvoir, il considérait la politique comme son chemin naturel. Il s'y est jeté avec volupté, avec passion. Les marches du pouvoir ne l'intimidaient pas. Il avait l'intention de les escalader quatre à quatre, et c'est ce qu'il a fait » (sur sa jeunesse vichyste, PA, p. 102).

ou celle de l'escalade :

« C'est cependant au moment où le PC se comporte de plus en plus comme un adversaire et où les Français /.../ lui préfèrent Michel Rocard que François Mitterrand, inlassable, entreprend son escalade décisive vers le pouvoir» (PA, p. 287).

La présidence, en tant que (haut) lieu prééminent, suscite la métaphore du cheminement. La vie de François Mitterrand, véritable anachorèse, est donc immanquablement racontée comme un parcours, un itinéraire, ou une trajectoire ascensionnelle. La métaphore spatiale est un des procédés de mise en récit qui introduit un surcroit de cohérence dans la biographie du personnage.

\section{Topométrie}

À une toponymie qui nomme l'institution présidentielle, à une topographie qui représente des dénivellations et trace des cheminements, s'ajoute une topométrie. La métaphore spatiale est alors utilisée pour mesurer des hauteurs :

«Les Français, dit Jacques Thibau, détracteur aigu, s'aperçoivent que la hauteur du personnage n'avait pas d'autre référent qu'une idée de soi dévorante et destructrice de tout » (LSS, p. 307).

Elle peut être également utilisée pour mesurer des grandeurs, chaque fois qu'est attribué ou réfuté l'adjectif grand pour qualifier la personne présidentielle, en évaluant l'étendue des compétences ou la profondeur des convictions : grand raccommodeur des déchirures sociales (ADA, p. 32), grand modernisateur de l'économie mixte (PA, p. 170), s'il ne s'est pas comporté en grand économiste, il s'est comporté en grand Européen (PA, p. 169). Un commentateur joue malicieusement sur cette métaphore qui effectue un transfert de sens, d'une qualité physique à une qualité morale :

« Tout petit déjà, voulait-il [Mitterrand] devenir plus grand que les autres ?» (ADA, p. 86). 
Ainsi la première figure de la prééminence présidentielle qui se dessine à travers l'usage des métaphores spatiales est celle de la hauteur. Les nombreuses expressions métaphoriques rassemblées autorisent à parler d'une métaphore systématique pouvant se réduire à un concept métaphorique : la présidence est en haut. Ce constat vient confirmer le rôle de ce que George Lakoff et Mark Johnson appellent des métaphores d'orientation ${ }^{9}$. Cependant, toutes les métaphores rencontrées ne font pas système avec le concept métaphorique de hauteur. Notre corpus révèle une seconde métaphore : celle de la centralité.

\section{Le centre}

\section{Toponymie}

Comme pour les métaphores de la hauteur, celles de la centralité sont utilisées par les commentateurs pour nommer la présidence. Selon une métonymie courante, la présidence de la République est désignée par le bâtiment qui l'abrite. Le lieu est alors qualifié de central :

« Dès qu'on touche à l'Élysée, les interrogations et les interprétations fleurissent. Comment en irait-il autrement dès lors qu'il s'agit du lieu central du pouvoir ou supposé l'être » (ADA, p. 116).

«L'Élysée (comme, je suppose, la Maison Blanche, le Kremlin, ou tout autre lieu d'organisation des mirages centraux) : une lanterne magique améliorée par l'ingénierie télévisuelle /.../ / (LSS, p. 375).

\section{Topographie}

Si la toponymie fait de l'Élysée un centre, les commentateurs, lorsqu'il s'agit de situer l'institution, dessinent une topographie qui place également le président en position centrale. D'abord pour ce qui concerne les lieux eux-mêmes :

« Mitterrand, en géopoliticien avisé, se trouve à la fois libre et protégé au cœur du palais » (à propos de la disposition des lieux à l'Élysée, ADA, p. 36).

9. Voir G. Lakoff, M. Johnson, op. cit., p. 24-31. 
«Dans une maison [l’Élysée] ordonnée autour et par le désir du Président, la plus petite baisse de baromètre de sa cote de popularité peut donner lieu à des états d'âme /.../ » (ADA, p. 251).

Mais cette topographie est aussi sociale. L'élément mis en relation avec le président n'est donc pas spatial mais humain. La métaphore de la centralité spécifie alors la nature du lien qui existe entre le président et son « entourage ».

« Il n'y avait pas de règle ou de méthode pour devenir son ami [de Mitterrand] mais des strates, des cercles, des ajouts, des cooptations, des hasards » (PA, p. 62).

« Un détail aurait dû me mettre la puce à l'oreille : dans ses cercles concentriques d'amis [de Mitterrand] de cinquante, trente ou dix ans, aucun "déraciné supérieur", grand universitaire, chercheur ou enseignant » (LSS, p. 323).

Si l'entourage du président est formé de cercles, c'est donc qu'il en est le centre :

« Accéder au premier cercle, c'est être finalement admis par les dessalés à dénigrer celui qui en est le centre, mais avec des sourires entendus, en petit comité et selon certaines clés » (LSS, p. 553).

\section{Topologie}

Cette topographie, à la fois spatiale et sociale, se fait topologie lorsque la métaphore du cercle ou du centre est explicitement associée à un mode de connaissance :

«Pour comprendre la vie quotidienne à l’Élysée, il est nécessaire de décrire les cercles - Mitterrand est un homme de cercles, pas concentriques mais séparés - qui constituent son entourage au palais. Il a placé là des personnes qui lui sont utiles pour des tâches très différentes, à des moments très particuliers. Comme dans sa propre vie, le système est très compliqué. Lui seul possède le plan d'ensemble » (ADA, p. 42).

«Au fur et à mesure /.../ s'est bâti aussi à l'Elysée un monde clos régi par un seul homme qui, seul, détient toutes les clefs » (ADA, p. 38).

Placer le président au centre, c'est donc encore une autre manière de dire qu'il est prééminent. Mais, entre la métaphore de la hauteur et la métaphore du centre, y a-t-il cohérence et compatibilité ? 


\section{Le haut et le centre}

Les métaphores de la hauteur et de la centralité peuvent se trouver réunies dans un même énoncé, comme dans cette critique d'un président protecteur des malhonnêtes où le protecteur est au-dessus de ceux qu'il protège :

«Les profiteurs et les traficoteurs tournent toujours autour du pouvoir comme des mouches, et François Mitterrand avait le don de les attirer sous son aile » (VHM, p. 39).

La topographie institutionnelle qui fait correspondre configuration des lieux et hiérarchies sociales mêle également le haut et le centre :

«Ainsi va la ronde des rumeurs attisées par le désir permanent de pouvoir se faire connaitre et reconnaitre par le Président. En bas, le peuple des conseillers disséminés dans des ailes du bâtiment et dans la rue de l'Élysée. En haut, proche du paradis et jouxtant le cœur élyséen, celles et ceux qui ont directement accès au Président » (ADA, p. 52).

Cette co-occurrence des métaphores de la hauteur et du centre pose problème ici puisqu'il y a incohérence logique entre l'idée de hauteur et l'idée de profondeur présente dans cœur. Mais l'incohérence logique n'empêche pas la cohérence métaphorique car les deux métaphores, celles de la hauteur comme celle du centre, convergent pour éclairer un seul aspect de la fonction : la prééminence. Celle-ci ne peut se dire que de manière relationnelle : le haut s'oppose nécessairement à un bas, et le centre à une périphérie. Les deux métaphores, du haut et du centre, entretiennent donc entre elles une stricte homologie. Le procédé stylistique d'accumulation amène à penser que les deux métaphores - par « superposition »-traduiraient une même représentation de l'institution présidentielle centrale-prééminente. Le président n'est donc pas seulement celui qui est en haut, il est aussi au centre et on pourrait même ajouter que c'est parce qu'il est au centre qu'il est tout en haut.

Cette représentation centrale-prééminente trouve sa traduction lexicale dans une image qui joint l'idée du haut à celle du centre selon une cohérence à la fois métaphorique et logique : celle du sommet. En effet, si on parle des rencontres entre chefs d'Etat « au sommet », c'est parce que chacun d'entre eux occupe le « sommet de l'État». Il n'est donc pas surprenant de retrouver cette métaphore dans notre corpus, associée par exemple à la description topographique d'un cheminement : 
«Entre autres révélations dans le livre de Pierre Péan, il y a celle d'un jeune homme pétri d'orgueil, désireux de pouvoir et empli de la certitude d'arriver un jour aux plus hauts sommets de la responsabilité » (ADA, p. 203).

Le mot sommet désigne le lieu le plus élevé de certaines choses et s'applique de manière usuelle - mais non exclusive - aux réalités géologiques (montagne, rocher, pic). La métaphore du sommet fait système avec celle de l'escalade (déjà rencontrée précédemment) pour assimiler l'État à une montagne dont le point culminant serait la présidence. Ainsi les deux tomes de la biographie de François Mitterrand par Jean Lacouture ont pour sous-titre «Les risques de l'escalade » pour l'avant 1981 et «Les vertiges du sommet» pour les deux septennats. On remarquera que la distribution des pluriels et des singuliers dans ces deux expressions fait de la présidence de la République le (seul et unique) sommet culminant.

Cette métaphore du sommet se retrouve elle aussi associée, de manière surprenante pour l'analyste mais sans que cela choque le lecteur, à celle du centre :

«Et pourtant, comment justifier, au plus haut sommet de l'État, au cœur même de l'Élysée, l'écoute de journalistes, écrivains, hommes politiques » (à propos des écoutes téléphoniques, ADA, p. 29).

Cette juxtaposition de métaphores ne s'interprète pas de manière univoque. Correspond-elle à une équivalence ou à une complémentarité des deux images, celle du sommet et celle du cœur ? D'un point de vue strictement géométrique, il y a contradiction puisque sommet désigne prioritairement une prééminence sur un plan vertical tandis que cœur renvoie à l'idée de profondeur. Mais cette incohérence logique ne nuit pas au procédé métaphorique. Les deux images se complètent car celle du sommet, comme celle du cœur, comportent toutes deux l'idée d'un centre. En effet, par la métaphore qui assimile l'État à une montagne et la présidence à son sommet, le point culminant est aussi un centre : le sommet d'un cône est aussi bien, par projection sur le plan vertical, le sommet d'un triangle que le centre d'un cercle, par projection sur un plan horizontal. Le président, au sommet de l'État, est bien en haut et au centre.

Cette cohérence logique entre la métaphore de la hauteur et celle du centre permet une systématicité de la métaphore du sommet qui peut alors être développée de manière tout aussi cohérente pour caractériser les attributs propres à l'occupant des lieux : il bénéficie d'un panorama à 360 degrés. C'est ce que sous-entend Régis Debray lorsqu'il évoque le rôle attendu du président : 
« D'où ce paradoxe : l'accès d'un responsable de parti au poste suprême, qui devrait dégager les perspectives et dont on attendrait des vues plus élevées sur la condition humaine, suscite chez le nouvel élu un inexorable rétrécissement des horizons /... / (LSS, p. 317).

Cette représentation d'une prééminence selon deux plans - vertical et horizontal - n'est pas nécessairement propre à l'institution présidentielle mais semble caractériser le politique en général. Ainsi, aux origines du mot politique, on trouve une racine indo-européenne dont l'idée générale est celle d'un lieu élevé protégé par une enceinte. En tant qu'espace clos, cette enceinte délimite un extérieur, et un intérieur associé à l'idée d'un centre. Parallèlement, le berg ou burg des langues germaniques, proche de polis par le sens, évoque lui aussi une position élevée qui avantage le défenseur par rapport à l'assaillant. Bourg deviendra le lieu politique par excellence, et encore plus explicitement en anglais, la circonscription-type (borough). Ce qui fait dire à Odon Vallet que « le mot politique induit une double dimension, verticale et horizontale » provenant des impératifs de la défense et de l'échange, et qui correspondent, pour Maurice Tournier, aux deux dimensions contradictoires de la réalité sociale que sont le consensus et le conflit ${ }^{10}$.

Cette généalogie du mot politique vient éclairer quelque peu différemment la métaphore du sommet de l'État. Les dimensions verticale et horizontale de la prééminence présidentielle sont-elles toujours convergentes et univoques ? Si le président est représenté en haut et au centre, préside-t-il de la même manière lorsqu'il est en haut et lorsqu'il est au centre ? Ces deux positionnements se confondent-ils dans une même figure de l'institution ou correspondent-elles à des modes d'actualisation différents du rôle ? Plus précisément, et en respectant le domaine de validité que nous autorise notre corpus, les métaphores de la hauteur et du centre entretiennent-elles systématiquement une cohérence métaphorique?

La réponse dépend du contexte. On a vu précédemment que dans le jeu de langage de la topographie institutionnelle, lorsqu'il s'agit de situer le président dans un espace socialement signifiant, les métaphores de la hauteur et du centre ne sont pas compatibles (logiquement) mais peuvent être cohérentes ${ }^{11}$, en ce sens qu'elles viennent éclairer un même

10. O. Vallet, «Aux origines de politique » et M. Tournier, «Autour de politique : bourg et faubourg », Mots, $\mathrm{n}^{\circ}$ 18, mars, 1989, p.106-109.

11. Selon G. Lakoff et M. Johnson, les diverses structurations métaphoriques d'un concept visent des objectifs différents, en mettant en valeur différents aspects du concept. Si bien que la compatibilité complète entre les métaphores est rare tandis que la cohérence est un phénomène fréquent. Sur la différence entre cohérence logique et cohérence métaphorique, voir G. Lakoff, M. Johnson, op. cit., p. 104. 
aspect de la fonction présidentielle. Mais les métaphores spatiales ne servent pas seulement à nommer, situer ou mesurer, elles peuvent aussi être utilisées pour critiquer. Dans ce contexte, la cohérence entre les métaphores de la hauteur et du centre ne va plus de soi. L'entourage du président, par exemple, tombe souvent sous la critique de la «société de cour » :

« Il [le Dr Tarot] a eu une vision du palais désastreuse et s'est très vite fait sa petite idée sur le fonctionnement courtisan de certains autour du Président» (ADA, p. 277).

« Je me suis toujours demandé comment le Président avait accepté de vivre au milieu de cette ménagerie où tout le monde avait les doigts pleins de confiture » (à propos des « hommes d'argent», VHM, p. 40).

La métaphore dessine un mode de prééminence particulier en spécifiant le type de relation que le président entretient avec ceux par rapport auxquels il est situé. Les commentaires de Régis Debray viennent tout à la fois illustrer et expliciter cette hypothèse :

« Notre démocrate est un Valois : de la chambre à l'anti-chambre, du bureau au boudoir, le sérail est omniprésent et sans détour. Cela s'appelle "personnaliser les rapports". En fusionnant service et allégeance, il attise le psychodrame alentour ; et dans la nébuleuse appelée entourage, la course aux places s'aiguise en course à la proximité » (LSS, p. 330).

Dans cette critique, les relations personnelles qu'entretiennent l'entourage et les proches avec le président s'opposent à la transparence et à l'impersonnalité des collaborateurs d'un homme d'État. Derrière l'institution garante des intérêts de la Nation, il y a des liens personnels qui servent les intérêts particuliers d'une coterie. On retrouve ici les critiques qui s'exercent sur la cité domestique depuis la cité civique ${ }^{12}$. En effet, le lien civique entre les personnes (comme, par exemple, la représentation politique) se définit précisément comme un affranchissement par rapport aux relations de dépendance personnelle. D'une manière générale, les métaphores du cercle et du centre servent à qualifier un type de relation qui renvoie à l'univers domestique, par opposition à l'univers civique qualifié par les métaphores de la verticalité :

« Ce petit cercle était trop professionnel, et déjà trop près des allées du pouvoir pour faire chapelle /... / L'absence de discriminant hiérarchique prévient les vexations, les jalousies importantes; la communauté des illusions fait celle des individus » (à propos des collaborateurs du futur président, LSS, p. 315).

12. Voir le «tableau des critiques » dans L. Boltanski, L. Thévenot, Les économies de la grandeur, Gallimard, 1992, et plus particulièrement la critique du monde domestique depuis le monde civique, p. 308 et suiv. 
L'image du cercle - plan horizontal - s'oppose à l'échelle hiérarchique - plan vertical. Entrer dans le cercle, c'est déjà se placer auprès du centre :

« Je fus, entre 1981 et 1988, du deuxième cercle, de " ceux qui rencontrent le chef de l'État quand ils le désirent et qui peuvent lui parler un peu de tout". Les "commandos", 1' "escadron volant", les "mousquetaires", disait l'échotier, à bon escient. Situés hors hiérarchie, les "chargés de mission auprès" avaient l'accès direct mais erratique » (LSS, p. 300).

Les images de la hauteur et du centre n'entretiennent plus de cohérence ni métaphorique ni logique. Le contexte sémantique les oppose et elles viennent éclairer chacune un aspect différent de l'institution présidentielle.

On a pu constater les effets de réalité propres à la fiction constitutionnelle qui place le président de la République au-dessus des partis. Le lexique de la hauteur relevé dans ce corpus de récits du personnage présidentiel témoigne de la prégnance de ce mode de représentation de la prééminence présidentielle, qui fonctionne alors comme la réitération et la ratification d'un ordre social et politique. Mais ce serait un parti pris de mauvaise méthode que de réduire ces commentaires à un simple reflet de la figure dessinée par le texte constitutionnel et ses interprètes. Les textes que nous avons pris pour objet montrent que cette mise en forme de la prééminence présidentielle à travers la métaphore spatiale s'effectue selon une pluralité de principes et de registres dont il faut rendre compte pour comprendre comment se construit, en contrepoint d'une figure présidentielle placée " en haut» de l'édifice civique, une autre figure qui place le président « au centre » et le fait ressembler à une sorte de patriarche. Reste à savoir si cette dernière image du président est propre à la mise en scène mitterrandienne du pouvoir, reprise et amplifiée par ses commentateurs, ou si elle est le résultat d'une grammaire constitutive de ce singulier exercice de l'autorité qu'on appelle présider. 